\title{
Energy-Efficient Boundary Detection for RF-Based Localization Systems
}

\author{
Tsung-Han Lin, Student Member, IEEE, Polly Huang, Member, IEEE, \\ Hao-Hua Chu, Member, IEEE, and Chuang-Wen You, Student Member, IEEE
}

\begin{abstract}
Boundary detection is a form of location-aware services that aims at detecting targets crossing certain critical regions. Typically, a lower location sampling rate contributes to a lower level of energy consumption but, in the meantime, delays the detection of boundary crossing events. Opting to enable energy-efficient boundary detection services, we propose a mobility-aware mechanism that adapts the location sampling rate to the target mobility. Results from our simulations and live experiments confirm that the proposed adaptive sampling mechanism is effective. In particular, when experimented with realistic errors measured from a live radio-frequency-based localization system, the energy consumption can be reduced significantly to 20 percent.
\end{abstract}

Index Terms-Boundary detection, energy efficiency, indoor localization.

\section{INTRODUCTION}

L OCATION-AWARE applications [1] are coming of age and to $\omega$ be realized in many everyday scenarios, for instance, asset tracking in warehouses, patient monitoring in medical facilities, and household management at home. These applications typically provide different types or levels of services based on the location information, supplied by localization systems. Much recent localization research [2], [3], [4], [5] concentrates on improving the accuracy of pinpointing the physical location of the target.

In this work, we focus on a class of location services that aims at detecting the crossing of boundaries, instead of tracking mobile targets constantly. For example, enemy tanks crossing the border line, expensive jewelries exiting the shop, or young children entering the balcony. These services do require as early detection of the target crossing boundaries as possible. That is, the users would prefer being notified of the boundary crossing events before the target goes too far into the critical region. Frequent sampling of the location information is typically the solution to allow accurate detection of the boundary crossing events.

However, energy efficiency of the mobile units, typically small, battery-powered tags or badges for better

- T.-H. Lin is with the Department of Electrical Engineering, National Taiwan University, BL-621, No. 1, Sec. 4, Roosevelt Rd., Taipei 106, Taiwan, R.O.C. E-mail: b90901046@ntu.edu.tw.

- P. Huang is with the Department of Electrical Engineering, Graduate Institute of Communication Engineering, and Graduate Institute of Networking and Multimedia, National Taiwan University, BL-613, No. 1, Sec. 4, Roosevelt Rd., Taipei 106, Taiwan, R.O.C.

E-mail:phuang@cc.ee.ntu.edu.tw.

- H.-H. Chu is with the Department of Computer Science and Information Engineering and Graduate Institute of Networking and Multimedia, National Taiwan University, Room 518, CSIE Building, No. 1, Sec. 4, Roosevelt Rd., Taipei 106, Taiwan, R.O.C. E-mail: hchu@csie.ntu.edu.tw.

- C.-W. You is with the Department of Computer Science and Information Engineering, 7F-5, No. 143, Sec. 1, Keelung Rd., Taipei 110, Taiwan, R.O.C. E-mail: you@ubitus.net.

Manuscript received 21 Dec. 2006; revised 15 Dec. 2007; accepted 12 May 2008; published online 2 June 2008.

For information on obtaining reprints of this article, please send e-mail to: tmc@computer.org, and reference IEEECS Log Number TMC-0341-1206.

Digital Object Identifier no. 10.1109/TMC.2008.84. wearability, attached to the tracked target is essential to the usability of the system. The energy consumption of a mobile unit is proportional to the sampling rate of the location information-the rate at which the localization infrastructure and its counterpart mobile units are triggered to perform the necessary communication and computation.

The dilemma with fixed-rate sampling is that the sampling rate can be set high to provide real-time location information, but when the target is far from the critical region where the timely service requirement is not as high, the high sampling rate will be unnecessary and there is no need for such waste of energy. Aiming at improving the energy efficiency and boundary detection accuracy at the same time, we propose an adaptive location information sampling mechanism based on the location and predicted mobility of the target.

Given the critical region to watch closely from the application, the mechanism controls the rate the localization system is triggered to acquire location information. When the target moves fast or close to the critical region, the sampling rate increases to enable accurate detection of the target entering the critical region. When the target slows down or moves away from the critical region, the sampling rate decreases to conserve energy. This mobility-aware adaptive sampling mechanism can be coupled with general radio-frequency (RF)-based localization systems and the integrated mobility-aware localization systems can work transparently for boundary-detection applications. The system architecture and interfaces are depicted in Fig. 1.

We propose two adaptive location sampling schemes for effective and energy-efficient detection of boundary crossing events. In the basic scheme, the system assumes the target is moving in constant speeds in between two location readings and estimates the velocity by two recent location samples. The estimated velocity is used to approximate the time the target will come near the boundary, which is used to wake the tag up to acquire the location information. In the extended scheme, the mobile tag incorporates a lowpower accelerometer to detect sudden changes of motion 


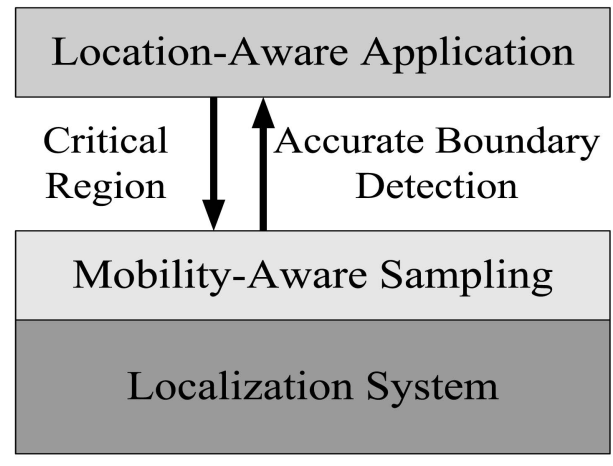

Fig. 1. System architecture.

which relaxes the constant-speed assumption in the basic scheme. It allows the tag to stay off when the target is in a stationary state and to quickly reestimate the target's velocity when there are sudden movement changes.

We evaluate the mobility-aware adaptive sampling mechanism by simulations and live experiments. The first set of simulations, assuming no localization error, demonstrates the full potential of mobility-aware sampling to fixed-rate sampling. The second set, applying measured localization error from RF-based localization systems, shows that the benefit of mobility-aware sampling, although still significant, is compromised by localization errors. The mobility-aware adaptive sampling mechanism reduces energy consumption to 5 percent in the withoutlocation-error case and 20 percent in the with-location-error case. The live tests echo the simulation results.

Our contribution is twofold. First of all, we propose a mobility-aware adaptive sampling enhancement for boundary detection in RF-based localization systems. Similar ideas of trading location fidelity for better energy efficiency have also been proposed in other localization systems [6], [7]. These works will further be discussed in Section 5. Second, with experiments, we validate that the mechanism is able to improve the energy efficiency when maintaining the boundary detection error at the same level.

The remainder of this paper is structured as follows: Section 2 gives an overview of the mobility-aware adaptive sampling mechanism. The mechanism is shown promising with thorough simulations in Section 3. Section 4 describes our implementation and the results from the live tests. In Section 5, we review the work in localization and mobile systems that are relevant to this study. And finally, we conclude and outline the work to pursue in the future.

\section{Mobility-Aware Sampling Mechanism}

The objective of the adaptive sampling mechanism is to sample right on the time the target coming to the critical point as illustrated in Fig. 2a. The key to capture the time the target comes near the critical region is the accuracy of predicting the mobility of the dynamic target. We present, in this section, the two schemes used to predict the target mobility. Assuming the target moves in constant velocity, in the basic scheme, we estimate the velocity of target by recent location readings. In the extended scheme, we relax the constant-velocity assumption, by adding an accelerometer to the tag to detect sudden change of velocity and trigger resampling of the target's location upon detecting such an event.

\subsection{Basic Scheme}

We assume the target moves in constant velocity in the basic scheme. Observe Fig. 2a. P1 and P2 are the two most recent sample points. The velocity is estimated by dividing the vector P1 to P2 to the time between the two points, shown as

$$
V=\left|\vec{P}_{2}-\vec{P}_{1}\right| /\left(t_{2}-t_{1}\right) .
$$

The critical point $C$ is where the line of movement intersects with the critical region. As the target arrives at position $P_{2}$, the system sets the time for the next sample by calculating the time for the target to move from the current position $P_{2}$ to the critical point $C$ in velocity $V$. Note that if the critical region is in irregular shape, it may take some computation to obtain the distance between current location and the critical region. Denoting the distance between $P_{2}$ and $C$ as $D$, the expected time elapsed $T$ for the object to move across the critical points can be computed by

$$
T=D / V .
$$

To avoid drastic error due to very rough estimation of velocity, we further bound the maximum value of the

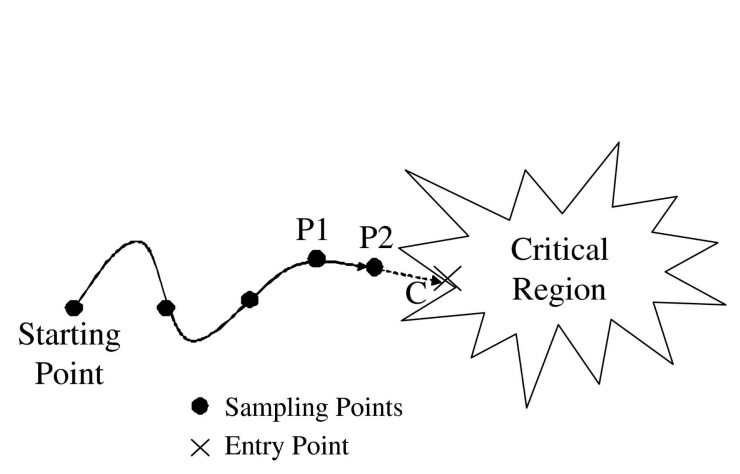

(a)

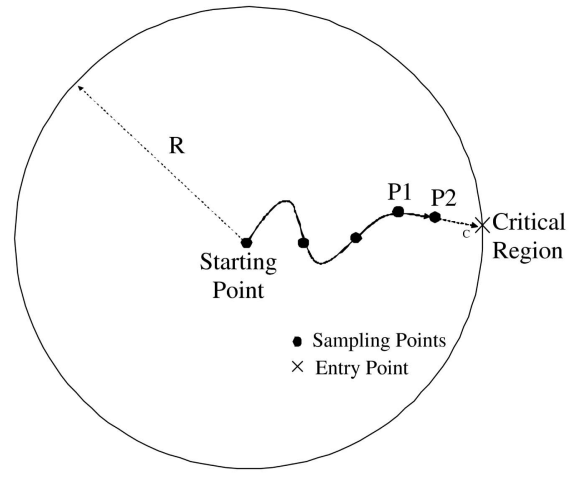

(b)

Fig. 2. Illustration of mobility-aware sampling. (a) General mechanism. (b) Simulation setting. 
sampling interval. This upper bound limits the error of detecting the target crossing the critical region's boundary. For example, the sampling interval is set to the upper bound instead of infinity to prevent from a false prediction of the target moving in zero velocity. The smaller the upper bound is, the more likely the system will be triggered to localize the target at the critical point. However, it will also result in a smaller amount of energy conservation.

In practice, localization systems report target positions with errors. If two recent reports are taken within a very short period of time, the physical change of the target's position is relatively small to the localization error. In other words, the velocity prediction will be dominated by the location estimation error. As a result, more frequent sampling will not help improve the accuracy in detecting the boundary crossing events. Setting a lower bound avoids such ineffective use of energy.

\subsection{Extended Scheme}

The assumption of the target moving in constant velocity is strong. In general cases, the target moving in the space is very likely to make a sudden change of velocity. Consider the extreme case where the target begins to move from being static. The chance is high that the target crosses the boundary way before the time has arrived to sample the next location. Setting of an upper bound limits the error of detecting boundary crossing events. However, the trade-off of setting the upper bound large or small is the same in the basic scheme.

In order to capture these sudden changes of velocity without setting a small upper bound, we add an extra sensor to help infer the mobility. By using a cheap and lowpower 2-axis accelerometer module, a clear difference between the stationary and the mobile period can be observed. When the target is moving, the sensor readings show a big variance; when the target stays static, the readings remain stable. Therefore, this 1-bit indicator can be obtained by examining whether the variance exceeds a certain mobility threshold over a preset time window.

With this indicator, we can suppress frequent and unnecessary sampling during the stationary period. Once the target starts to move, the accelerometer can capture the motion immediately and trigger the tag to operate using the basic scheme. Note that when the system returns to the basic scheme mode, it will set the next sampling interval to minimum in order to obtain an estimate of the new velocity.

\section{Simulation}

The objective of the simulation is to demonstrate quantitatively the benefit of mobility-aware adaptive sampling mechanism, in particular, how much the energy efficiency and the accuracy of the critical point localization can be improved by adapting to the predicted velocity. We implemented the fixed-rate mechanism, basic scheme, and the extended scheme for comparison. Our evaluation consists of two sets of simulations-one assuming no localization error and the other assuming localization error measured in a Zigbee radio signal strength indicator (RSSI)-signature-based localization systems [3]. The implementation of the localization system will be detailed in Section 4. Based on the simulation results, we discuss 1) the performance improvement of the basic and extended schemes relative to the basic fixed-rate mechanism, 2) how the benefit of mobility-aware mechanism changes taken into consideration localization errors, and 3) how the target mobility and boundary crossing rate affect the efficiency of the adaptive sampling schemes.'

\subsection{Simulation Settings}

The idea of mobility-aware adaptive sampling is applicable for general localization systems. In a 2D space, the shape of the critical regions could be irregular. Finding the critical points is not trivial. To focus more on evaluating the adaptive sampling mechanism, we consider a simplified setting. The critical region is only determined by the distance between the target and a reference point in the simplified version. Illustrated in Fig. $2 b$, regions more than $R$ distance away from the reference point are set as the critical region. The critical points compose a circle centered at the reference point. The amount of overhead required to perform one location update varies between different localization techniques. To simplify the problem, we assume that a fixed number of beacons are placed in the area, and the target node can obtain its location through one message exchange with the beacons.

Using the simplified setting, the mobility-aware adaptive sampling mechanism is implemented in the network simulator version 2, i.e., $n s-2$ [8]. $n s-2$ is a packet-level discrete event simulator for wired and wireless data network simulations. The radio range is set to $80 \mathrm{~m}$ while the area of experiment is set to $70 \times 70 \mathrm{~m}^{2}$ to ensure that the target and beacon nodes will be reachable to each other throughout the simulations. The range of the reference point to the critical point $R$ is set to $20 \mathrm{~m}$.

To simulate the movement of the user, we adopt the random waypoint mobility model [9]. In this model, nodes move with random speeds toward random destinations. A different pause time between two consecutive movements is set to represent a different degree of mobility. A lower pause time implies a higher overall mobility, i.e., a higher average speed and a higher frequency of changing directions. A number of more realistic, scenario-based mobility models are used in the testbed experiments described in Section 4. In our simulations, the speed of each movement is chosen uniformly random from 0.3 to $2 \mathrm{~m} / \mathrm{s}$, which is also around the speed range of human walking. We vary the pause time interval from 0 to 1,000 seconds. With different pause time, random moving speeds and destinations are generated to run an 8-hour simulation to cover the 9 to 5 working hours.

\subsection{Performance Metrics}

The performance metrics are detection accuracy and average location sampling rate. The application would prefer an accurate report when the target just arrive at the critical region. In our simulations, the location of the target is reported when the target is $R$ unit distance away from the reference point. Thus, the detection accuracy is defined as the distance between the reported position and the critical distance $R$. 


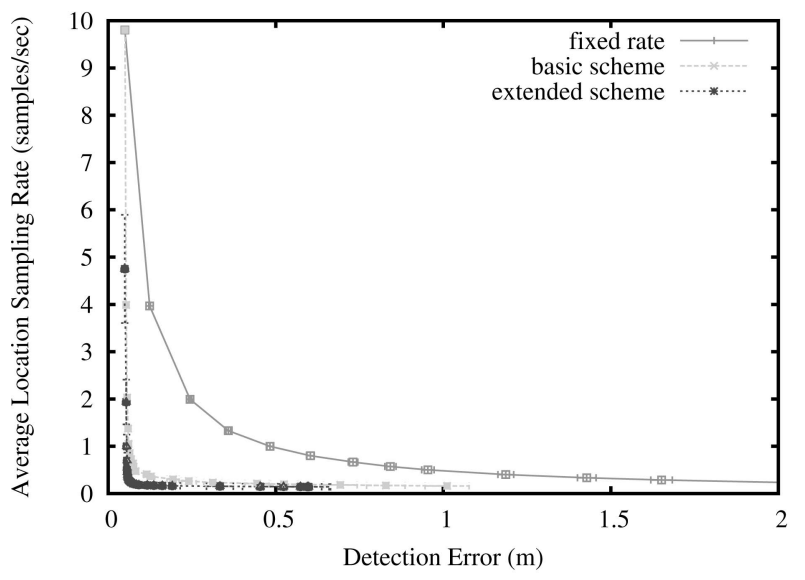

Fig. 3. Results without localization error.

From the simulations, we are not able to measure the total energy consumptions of the localization system. In fact, how much energy is required to localize the target depends on the exact design and varies from system to system. Here, we assume that given a localization system, the energy consumption per localization is constant and consider a minimum localization system. In that, a small fixed amount of nodes equipped with RF transceivers are placed in the area. The target equipped with the corresponding transceivers receives the RF signals to estimate its position from which the target velocity can be estimated and the next sampling time can be derived, using the mechanism defined in Section 2.1.

Consider the localization systems. Since the RF interfaces are the primary energy consumer, the number of localization samples directly reflects the energy consumption of the mobile target. The average location sampling rate is measured to evaluate the energy efficiency of the mechanism. A lower average sampling rate implies better energy efficiency. In the extended scheme, an extra accelerometer is incorporated. The power consumption of the additional accelerometer will be discussed in Section 4 .

\subsection{Results without Localization Error}

The simulation results with zero localization error is shown in Fig. 3. In the figure, the $x$-axis is the detection accuracy and the $y$-axis is the average location sampling rate. Every data point is plotted with 95 percent confidence interval. Because no error comes from the localization system, the error observed here is entirely due to sudden changes of movement.

The fixed-rate sampling results are drawn by simulations that each uses a different sampling interval, varying from 0.1 to 10.5 seconds, respectively. The data point of the 0.1-second interval lies in the upper left side of the plot, which has the smallest detection error and the highest location sampling rate. By increasing the sampling interval, the detection error would increase accordingly but the location sampling rate would decrease. Thus, the curve grows to the lower right side of the plot where the detection error is larger and the location sampling rate is lower. The curve shows that there exists a trade-off between the error and energy efficiency. The higher the accuracy requirement from the application, the more energy the system will consume.

The fixed-rate result also shows as a performance bound. Any effective mechanisms must produce error-energy curves that are to the lower left of the fixed-rate curve. These curves suggest that these effective mechanisms can achieve better detection accuracy given the same energy consumption in the fixed-rate sampling, or better energy efficiency for the same accuracy.

The other two lines show the results of tuning the upper bound of the basic and the extended schemes. We first fixed both the lower bound and the upper bound to 0.1 second. Then, we gradually varied the upper bound from 0.1 to 11.5 seconds for the basic scheme, and from 0.1 to 55.5 seconds for the extended scheme. The data point of the 0.1-second upper bound again lies in the upper left side of the plot, since a smaller upper bound can limit the detection error with a higher location sampling rate. The two lines also grow toward the lower right side of the plot by increasing the upper bound. We observe that the average location sampling rate drops rapidly at the beginning while the average detection error does not vary a lot. This shows that the mobility-aware mechanism effectively adjusts the sampling intervals to a slower rate to conserve energy and yet maintains a similar level of accuracy. After the initial drop, further relaxing the upper bound does not help too much in reducing the location sampling rate while the error increases significantly. This means that when the upper bound is too large, the system may fail to react to movement changes during the sampling interval.

With the help of the accelerometer, the average location sampling rate of the system is no longer bounded by the upper bound sampling rate. If the accelerometer indicates that the target is static, there is no need for the target to resample its location. The target can completely turn off its $\mathrm{RF}$ interface throughout the stationary period. The extended scheme curve, therefore, is shifted downward and saves more energy given the same detection error.

\subsection{Results with Localization Error}

The benefit we observe in the adaptive sampling mechanism is a result of exploiting the target mobility. Error in localization may impact the accuracy of the velocity prediction, and in turn, compromises the benefit of adaptive sampling. To evaluate the degree of impact the localization error may incur, we measured the localization errors from an actual Zigbee RSSI-signature-based localization system. The error distribution is obtained from 1,000 measurements in our localization system, which has the 50 percent percentile error at $1.69 \mathrm{~m}$ and the 80 percent percentile error at $2.99 \mathrm{~m}$. The trace is used to drive the simulator to study the impact of localization error.

We found that when the fixed sampling interval is set small enough, the detection accuracy of the RSSI-based localization system cannot be improved correspondingly while more energy is consumed. It does not follow the energy-accuracy trade-off in high sampling rate. This phenomenon is due to the significantly larger localization error, which limits the detection accuracy. When the location sampling rate is too high, the detection accuracy becomes dominated by the localization error instead of the 


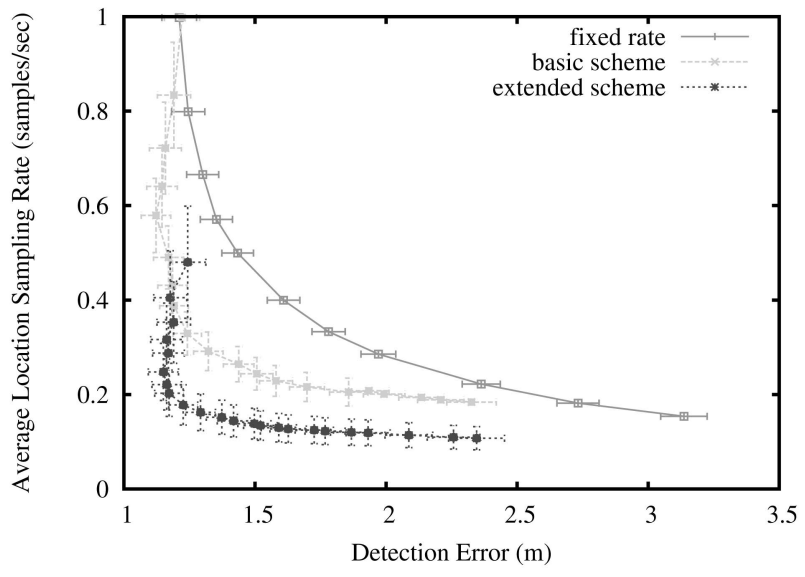

Fig. 4. Results with localization error.

sampling rate. The excessively small sampling interval can lead to energy waste. Therefore, in the mobility-aware mechanisms, the lower bound of sampling intervals should be set to the point where the detection accuracy saturates in order to avoid unnecessary sampling overheads. We empirically determine this saturation point as 1 second and set the lower bound accordingly in the simulations. We vary the sampling interval from 1 to 7.5 seconds for the fixed-rate sampling mechanism. For the mobility-aware sampling mechanism, the upper bound is varied from 1 to 15.5 seconds for the basic scheme, and from 1 to 35.5 seconds for the extended scheme.

The simulation results are shown in Fig. 4. Fig. 5 shows the results with and without localization error together for ease of comparison. Similar to the no-error case, on the upper left side of the figure is the data point with 1-second sampling interval. The curves grow toward lower right when the fixed sampling interval or the upper bound of sampling intervals increases. It can be seen that the range estimation error impacts the mobility-aware mechanisms. The two curves of the mobility-aware adaptive mechanisms are drawn closer to the fixed-rate curve. Even so, the resulting curves still fall to the lower left of the fixed-rate sampling mechanism, and the mobility-aware mechanisms show a qualitatively similar scaling property. Since the localization error of the RF-based system significantly degrades the accuracy of the velocity prediction, therefore, the adaptation on the sampling interval becomes less effective in achieving the optimal accuracy-energy combination. The extended scheme curve still shows better energy efficiency, since the motion prediction by the sensor is not affected by the extra localization errors.

\subsection{Gain of Mobility-Aware Sampling}

To clearly demonstrate the benefits of the mobility-aware sampling mechanism, we show the amount of location samplings performed relative to the fixed-rate sampling mechanism in Fig. 6. In this figure, the comparison is done by controlling the detection accuracy. The percentage of location samplings is computed as the average sampling rate of the mobility-aware mechanisms divided by the fixed-rate mechanism, which achieves the same detection accuracy. It can be seen that both the basic scheme and the

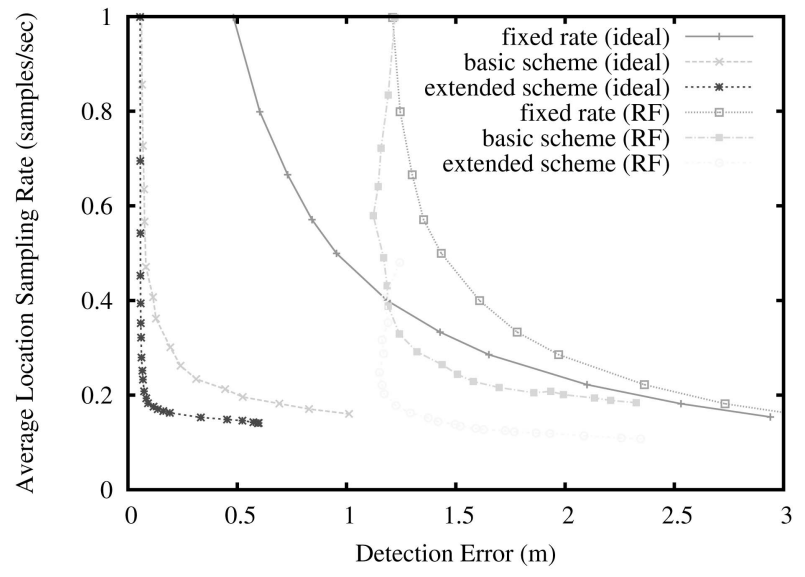

Fig. 5. Comparison of no-error and RF-error case.

extended scheme can achieve significant energy reduction. The sampling rate can be reduced to 8 percent in the noerror case and 39 percent in the RF-error case. With the assistance of the accelerometer, the sampling rate can be further reduced to 5 percent in the no-error case and 20 percent in the RF-error case.

Looking closer to the increasing trend of the sampling rate ratio as the detection error increases, one can also observe that the benefit of the adaptive sampling mechanism declines when the detection error requirement is loose. This is because when we want to trade the detection accuracy to save more power by relaxing the upper bound, the resulting larger sampling intervals exaggerate the detection error contributed by the error in velocity estimation. This also suggests that if an application can tolerate very large detection errors, the fixed-rate sampling mechanism can be a better choice. Note that the detection error is contributed by the untimely location sampling in addition to the inaccurate location estimation.

\subsection{Impact of Mobility}

Note that in the mechanism section, the sampling rate is set to the upper bound when the velocity equals zero. If an additional accelerometer is added, the location sampling can be simply stopped when the target is stationary. The mobility-aware sampling mechanisms are expected to

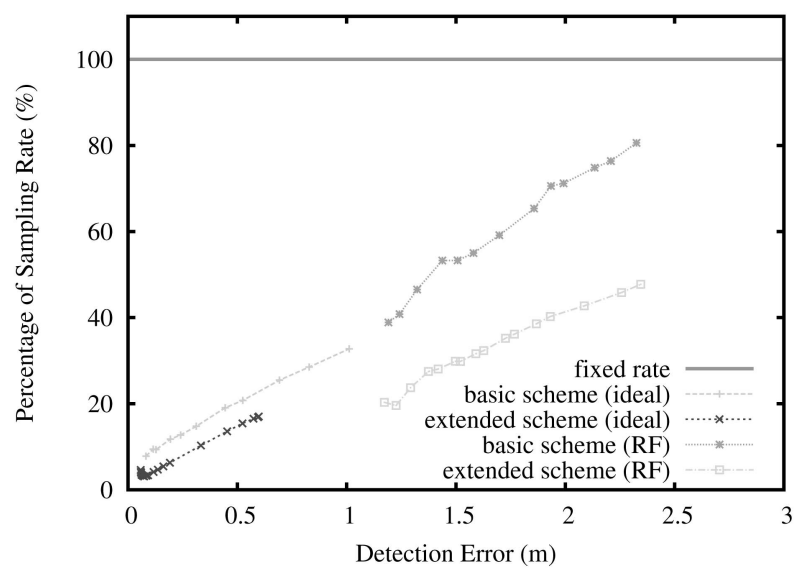

Fig. 6. Reduction of location sampling rate. 


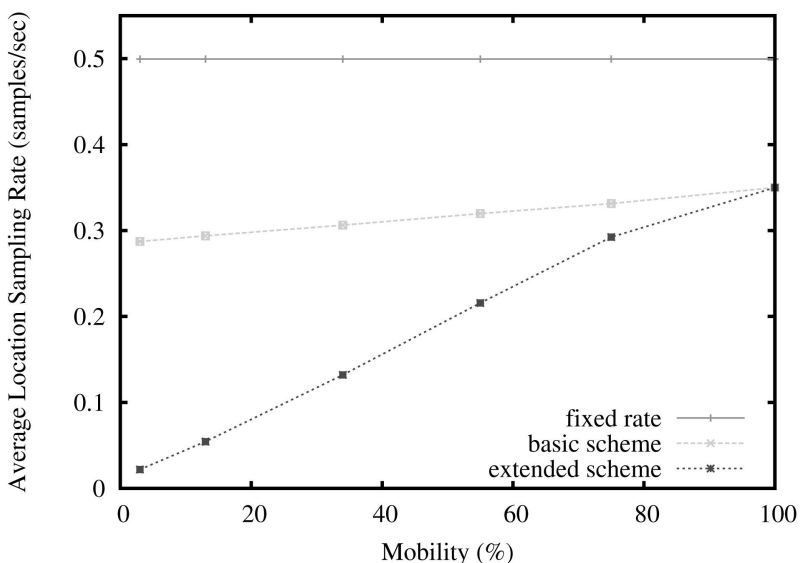

(a)

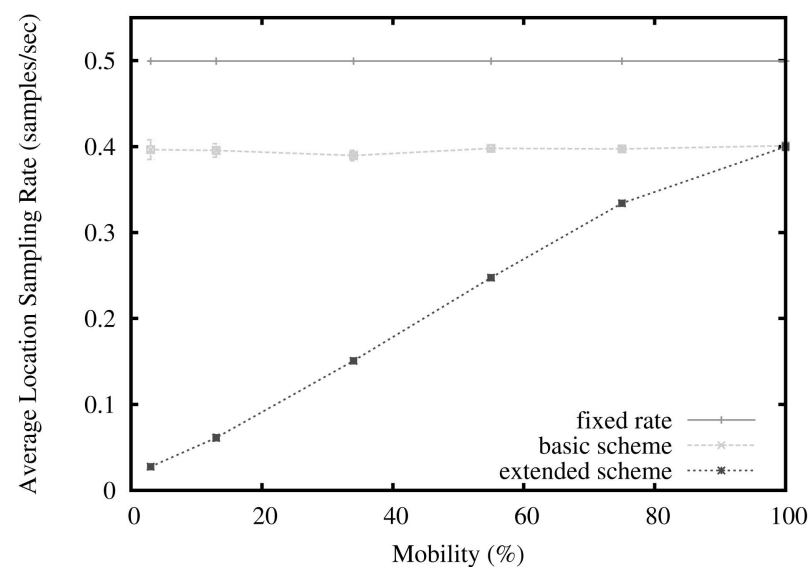

(b)

Fig. 7. Impact of mobility on sampling rate. (a) Without localization error. (b) With RF localization error.

conserve more energy when the mobility is low. The simulation results shown in Figs. 7 and 8 are the average location sampling rate and detection accuracy over various mobilities. The mobility here is defined as the percentage of time that the target is moving. We generate scenarios based on different percentage of moving periods to evaluate the impact of mobility. For example, pause time 1,000 seconds in the random waypoint model equals to 0.7 percent mobility in the experiments. All data points in Figs. 7 and 8 are plotted with 95 percent confidence interval.

For both no-localization-error case and RF-error case, we use the 1-second lower bound and 3.5-seconds upper bound setting for the mobility-aware mechanisms and 2-seconds sampling interval for the fixed-rate mechanism. This setting is close to the optimal setting with the largest energy savings in the RF-error case. We first show the relationships between the average sampling rate and mobility. The simulation results for the no-localization-error case are shown in Fig. 7a and the results for the RF-error case are shown in Fig. $7 b$. In Fig. $7 \mathrm{a}$, the average sampling rate decreases for both the basic and extended schemes as the mobility decreases. This shows that both mechanisms are able to adapt the sampling rate to target mobility. Without any localization error, the basic scheme is able to detect the stationary period, and switch the sampling rate to the upper bound to maintain the system reactivity. With the incorporation of accelerometer, the system can go to sleep for the whole stationary period. Once the target becomes mobile, the accelerometer helps trigger the system to return to the basic location sampling scheme. This advantage can be seen in the extended scheme curve, which has a steeper slope than the basic scheme because it does not need to check periodically even if the target does not move. When the target moves close to 100 percent mobility, there is very little margin for the extended scheme to work.

For the RF-error case, the simulation results are shown in Fig. $7 \mathrm{~b}$. We can see that the average sampling rate for the basic scheme is higher than that of the no-error case. The higher sampling rate again shows the effect of localization error, given that localization error gives a false perception to the adaptation mechanism that the target node keeps moving; therefore, the sampling interval is raised high and can hardly adapt to target mobility. But if an accelerometer is incorporated, the sampling rate again decreases rapidly. This is because the extended scheme captures the mobility

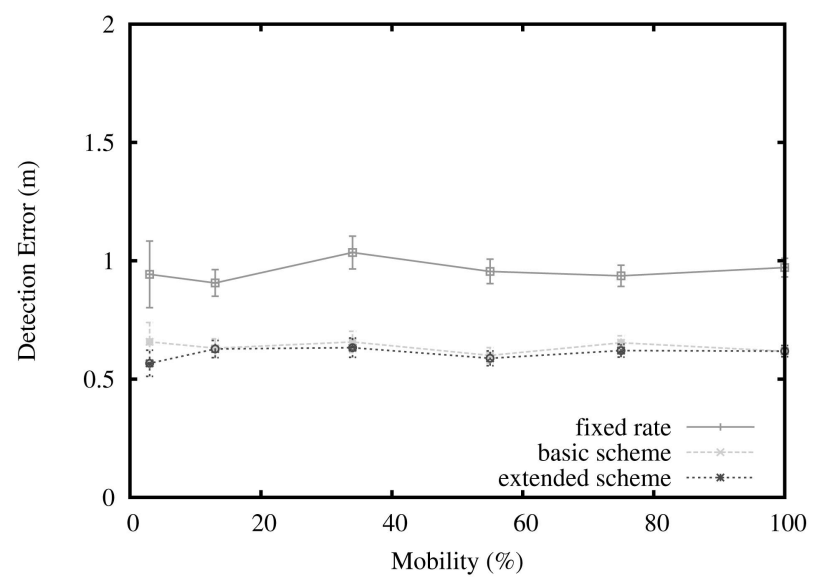

(a)

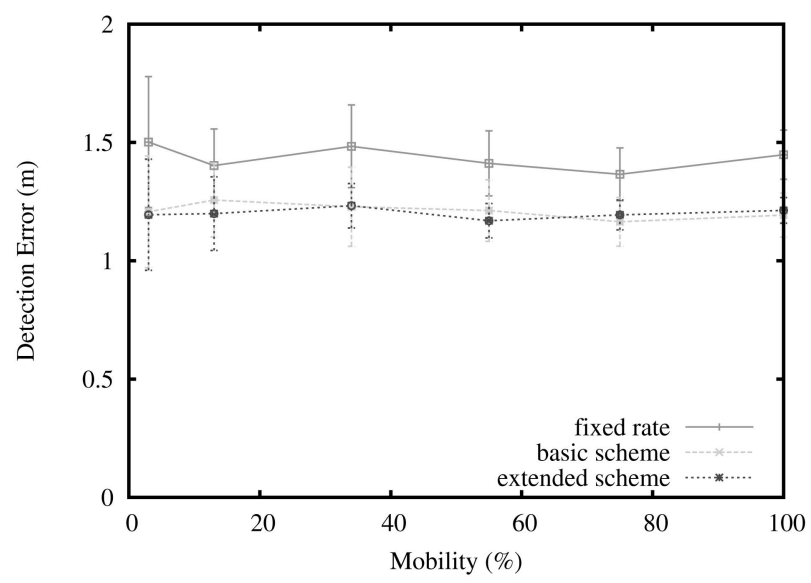

(b)

Fig. 8. Impact of mobility on detection accuracy. (a) Without localization error. (b) With RF localization error. 


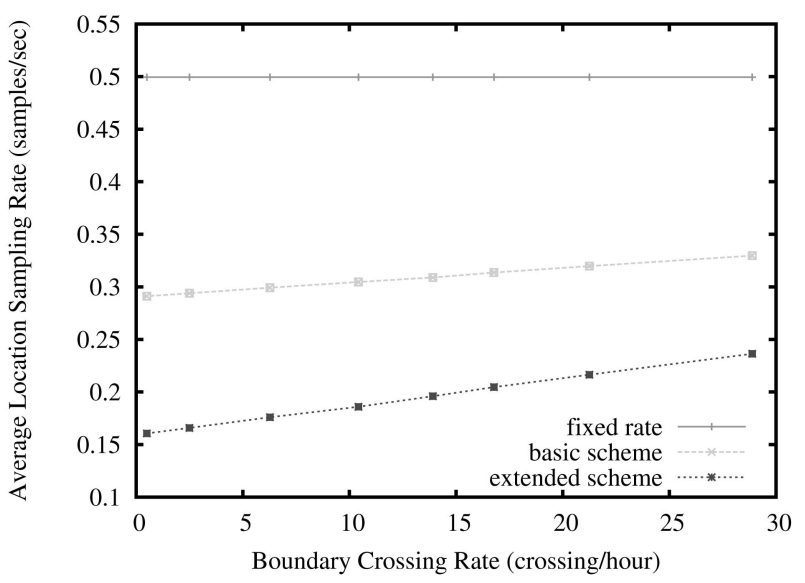

(a)

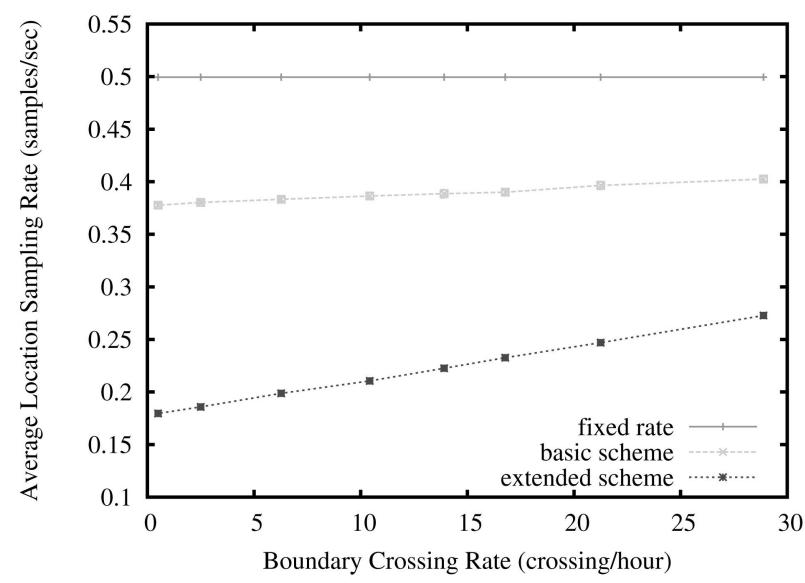

(b)

Fig. 9. Impact of boundary crossing rate on sampling rate. (a) Without localization error. (b) With RF localization error.

based on extra sensor readings, and the sampling rate is not affected by the localization error.

Fig. 8 shows the detection error with different mobility levels. In the no-error case (Fig. 8a), the errors of both mobility-aware mechanisms are similar and are also not affected by the mobility levels. This indicates that the upper bound of the sampling interval can maintain system reactivity in the zero velocity period. Also, setting the target node to sleep by the accelerometer can conserve more energy without introducing extra detection errors to the system. The errors in the RF-error case (Fig. 8b) show a similar trend, but the value is larger due to the localization errors.

\subsection{Impact of Boundary Crossing Rate}

In the mobility-aware sampling mechanism, the next sampling interval is determined by the predicted target velocity, as well as the distance to critical regions. In addition to target mobility, the rate of boundary crossing events can potentially impact the performance of mobilityaware mechanisms. If the target keeps moving across the boundary of the critical region, the average sampling rate will be raised higher by the adaptive sampling mechanism.

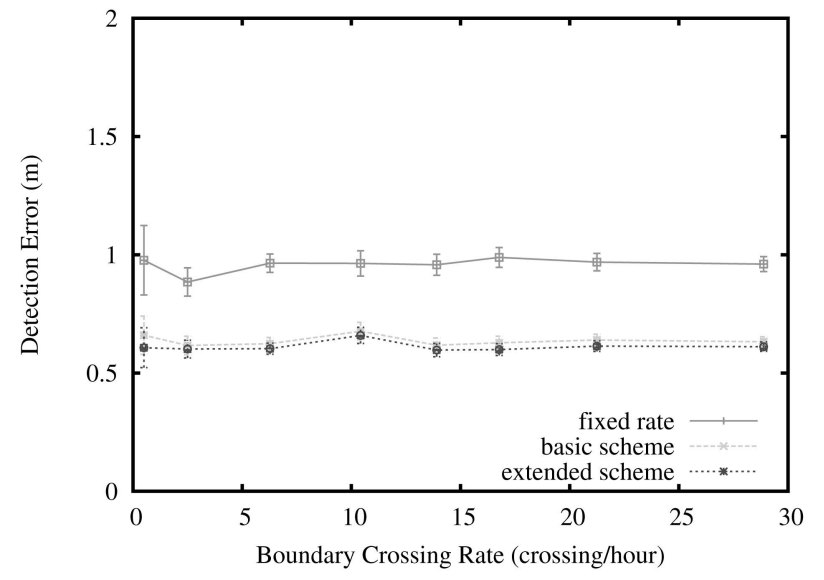

(a)
In this set of experiments, we vary the rate of boundary crossing events from 0.5 to 29 times per hour. We use the 75 percent mobility scenarios with 1-second lower bound and 3.5-seconds upper bound setting for the mobilityaware mechanisms and 2-seconds sampling interval for the fixed-rate. The simulation results are shown in Figs. 9 and 10 with 95 percent confidence interval. It can be seen that for both no-error and RF-error cases, the mobility-aware mechanisms achieve lower average sampling rates with similar detection accuracy when the boundary crossing rate is lower. This shows that the mobility-aware mechanisms can conserve energy not only when the target moves slower but also when the target stays far from the critical regions. Also, an offset of sampling rate exists between the basic and the extended schemes. This offset comes from the energy saved by the further reduced sampling rate in the stationary periods using the extended scheme.

\section{IMPLEMENTATION}

The mobility-aware adaptive sampling mechanism is further implemented and integrated to a localization system

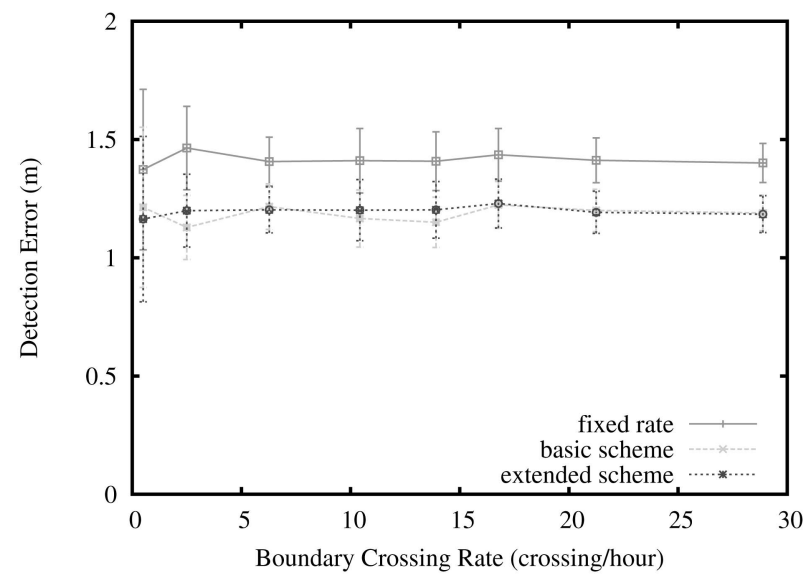

(b)

Fig. 10. Impact of boundary crossing rate on detection accuracy. (a) Without localization error. (b) With RF localization error. 


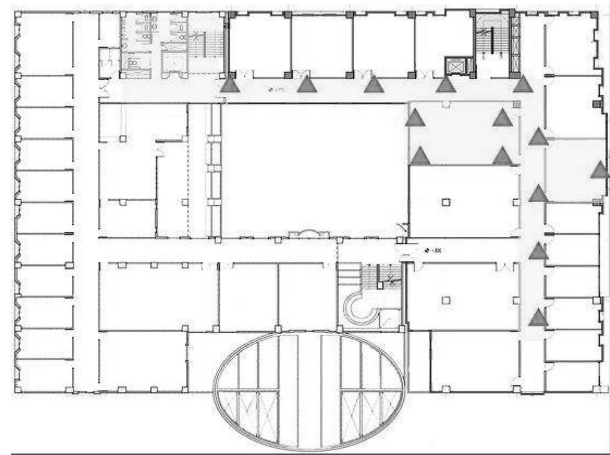

Fig. 11. Localization system.

to validate the simulation results. In this section, we describe how the testbed is set up and present the experimental results.

\subsection{System Implementation}

We set up a Zigbee-based localization system on the third floor of our department building, as shown in Fig. 11. An RSSI-signature-based approach, similar to MoteTrack [3], is adopted to estimate locations. The idea of the signature map is to exploit the mapping between a tag's location and the RSSI from a set of predeployed beacons, referred to as the RSSI vector. The tracking area is surveyed to construct the reference RSSI signature per sampled location. With the map, the system compares the collected RSSI vector to the reference RSSI signatures in the tracking phase to identify the closest possible location. With the map, the localization can be achieved by comparing the collected RSSI vector with the reference RSSI signatures to find out the closest possible location. We use the $k$-nearest-neighbor (KNN) method for signature comparison. In that, we select the top $k$ sample locations whose RSSI signatures are the closest to the collected RSSI vector. The KNN estimator would output a location as an average of the top $k$ locations' coordinates weighted by the euclidean distances between the RSSI vector and the signature. The location from the KNN estimator is later processed by particle filters [10], a nonlinear filter that incorporates human mobility models to improve the localization accuracy. During operations, the badge will turn its radio interface on to collect RSSI vectors and obtain an estimated location from the position engine. With the estimated location and accelerometer readings, the badge will determine a sleep interval based on the adaptive mechanisms and turn off its radio interface accordingly to conserve energy.

\subsection{Experimental Settings}

Our system consists of 14 Telos motes [11] deployed $6 \mathrm{~m}$ apart on the ceiling. These 14 motes serve as beacon nodes, periodically broadcasting messages containing RSSI values with a 200-ms interval. On the user side, the user wears a mobile badge composed by a MicaZ mote equipped with a 2 -axis accelerometer for localization and movement detection. Both Telos and MicaZ use the same 2.4-GHz Zigbee radio interface, and therefore can exchange $\mathrm{RF}$ messages between each other.

Six walking patterns with different mobility level ranging from 10 percent to 100 percent are designed to
TABLE 1

Scenario Representation of Mobility

\begin{tabular}{|l|l|}
\hline $\begin{array}{l}\text { Mobility } \\
\text { levels }\end{array}$ & Scenario description \\
\hline $100 \%$ & $\begin{array}{l}\text { A person strolled repeatedly from one end of } \\
\text { corridor to the other end. }\end{array}$ \\
\hline $90 \%$ & $\begin{array}{l}\text { A person walked along a corridor, entered } \\
\text { his/her office, briefly sat down to check } \\
\text { his/her calendar, and then went to a meeting } \\
\text { room. }\end{array}$ \\
\hline $70 \%$ & $\begin{array}{l}\text { A person walked along a corridor, bumped } \\
\text { into a friend, and stopped to chat for a short } \\
\text { time. }\end{array}$ \\
\hline $50 \%$ & $\begin{array}{l}\text { A person leisurely walked and browsed } \\
\text { through posters displayed on a wall along a } \\
\text { corridor. }\end{array}$ \\
\hline $30 \%$ & $\begin{array}{l}\text { A person walked to a meeting room to have } \\
\text { a discussion, occasionally moved to a white } \\
\text { board to explain an idea. but most of the time, } \\
\text { sat and listened to friends. }\end{array}$ \\
\hline $10 \%$ & $\begin{array}{l}\text { A person walked to a seminar room, sat and } \\
\text { listened to a long lecture. }\end{array}$ \\
\hline
\end{tabular}

allow performance comparison. Based on our experience moving in the building, a scenario is generated for each mobility level. Enlisted in Table 1 are rough descriptions of the moving patterns used in the experiments. Each scenario is about 15 minutes long with the walking velocity around $0.5 \mathrm{~m} / \mathrm{s}$. Since the RSSI values are vulnerable to environment changes, different rounds of experiments may not be comparable. To ensure fair comparisons, we configured the receiving tag to log every beacon packet containing the RSSI value along with the reception time throughout the experiment. The three sampling mechanisms were applied offline on the same collected traces to determine the received RSSI vectors and adjust the RSSI sampling rate. The location, therefore, can be estimated with the sampled RSSI vectors.

We set the critical region as a vertical line in the middle of the corridor to test the sensitivity of the sampling mechanisms. In each trace, there can be one to nine boundary crossing events based on different mobility. To avoid biased results, each trace is tested for 10 times with random start points. The sampling mechanisms should be able to detect each boundary crossing event accurately. We use the same parameter settings for the upper and lower bounds of the sampling intervals as in the simulations (1-second lower bound, 3.5-second upper bound for adaptive, and 2 seconds for fixed-rate) when conducting the live tests.

\subsection{Experimental Results}

As shown in Fig. 12, the results largely echo those of the simulation experiments in Section 3. For the fixed-rate and the basic scheme, the average sampling rate is similar regardless of the mobility. Although the basic scheme cannot adapt with target mobility due to the large localization error, the average sampling rate is still 75 percent to that of the fixed-rate mechanism. With the accurate detection of stationary period by the accelerometer, the average sampling 


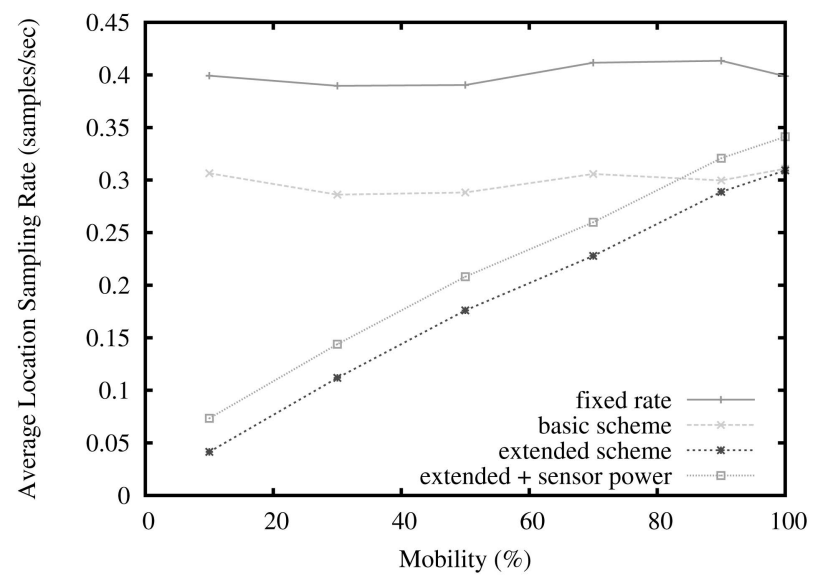

(a)

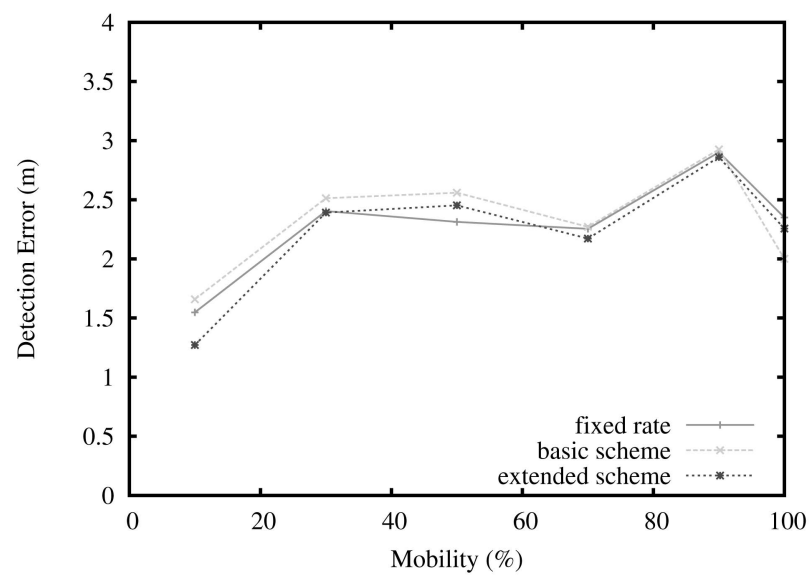

(b)

Fig. 12. Results from real localization systems. (a) Average sampling rate. (b) Detection error.

rate of the extended scheme drops significantly to 13 percent comparing to the fixed-rate mechanism in 10 percent mobility. Note that the average sampling rates of the three mechanisms are all lower than the results in the simulation section. This is because in a real localization system, it takes roughly 0.5 to 1 second for the receiving badge to collect enough RSSI values for a valid location estimation. The additional delay in effect increases the time interval between samples; thus decreases the average sampling rate. On the other hand, the detection errors of the three sampling mechanisms are all similar. This indicates that both the basic and extended schemes can save energy without sacrificing the detection accuracy, and outperform the fixed-rate sampling mechanism.

To take the additional power consumption drawn by the accelerometer into consideration, we compute the equivalent location sampling rate that uses the same amount of energy as the accelerometer for comparison. Table 2 shows the power consumption profile of the radio and accelerometer used in our experiments [12], [13]. Assume, to acquire each location sample, the radio needs to be put into the receive mode for 1 second to receive the RSSI vectors. The energy consumption for each location sampling is therefore $56.4 \mathrm{~mJ}$. Consider the worst case scenario that the accelerometer is always on without any power management. The energy consumed by the accelerometer for 1 second is $1.8 \mathrm{~mJ}$, equivalent to taking 0.032 location samples per second. The results after adding this equivalent location sampling rate are shown in Fig. 12a. The average location sampling rate of the extended scheme exceeds the basic scheme for mobility 90 percent and 100 percent. If the target moves

TABLE 2

CC2420 Radio and ADXL202JE Accelerometer Power Consumption

\begin{tabular}{|l|r|}
\hline Modes & Power consumption \\
\hline Radio Transmit & $52.2 \mathrm{~mW}$ \\
\hline Radio Receive & $56.4 \mathrm{~mW}$ \\
\hline Radio Idle & $1.28 \mathrm{~mW}$ \\
\hline Accelerometer & $1.8 \mathrm{~mW}$ \\
\hline
\end{tabular}

continuously, the extended scheme has very little effects and consumes more energy with the extra sensors. However, there exists some very low-power accelerometers which can help us minimize the additional sensor power consumption. For example, the power consumption of a more recent ADXL330 3-axis accelerometer [14] is $0.96 \mathrm{~mW}$, only half of the sensor used in our current deployment.

\section{Related Work}

The main contribution of our work is at the mobilityaware adaptive sampling of the location information based on the target mobility to improve both the boundary detection accuracy and energy efficiency. However, mobility-awareness and adaptive sampling are not new ideas in communication system research. There also exists a wide range of research introducing different localization techniques and improving the energy efficiency of wireless networks. In the following sections, we review and discuss, respectively, how this work is relevant or complimentary to the aforementioned research domains.

\subsection{Mobility-Aware Communication Systems}

The idea of using predicted mobility for overhead optimization has appeared in the literature of mobile and ad hoc networking computing. In mobile ad hoc networks, [15] controls the routing information updating rate efficiently by adapting the frequency to the distance between two nodes as well as node mobility. In the context of cellular PCS network, efficient paging [16], [17] is achieved by limiting the cell search to the region that the users are entering. For object tracking sensor networks, predicted object mobility is used to activate sensor nodes that are falling into the mobile object's proximity while the other nodes hibernate to conserve energy [18]. Limiting the region of communication or sensing, as proposed in the literature, reduces the system overhead in the spatial dimension. The mobility-aware adaptive sampling mechanism, complementary to the prior work, exploits the temporal dimension and adapts the sensing/sampling rate to the predicted mobility.

A recently proposed localization system [7] shares the same energy conservation objective as our proposed mechanism. The system reduces the amount of location 
data dissemination from the mobile unit to the location server by separating the tracking area into small predefined areas. Only when the mobile unit detects itself entering or leaving the targeted area, the location information is sent back to the central server. However, our work differs in that we optimize the energy consumption of the mobile units in using wireless interface to acquire location information. This is complementary to the zone-based system that aims at reducing the power consumption of using wireless interface to forward location data.

In other types of localization systems such as the vision-based systems, similar approaches in optimizing the location updating rate are introduced, because the cost to transmit pictures to obtain location updates is especially high in such systems. In [6], the system assumes target moves in constant speed and transmits a new picture to reestimate the location and moving direction when it predicts the target comes close to the corners in the space. The general idea is similar to our adaptive sampling mechanism but our extended scheme can further relax the constant-speed assumption and provide a more practical solution.

\subsection{Adaptive Sampling}

The idea of adaptive sampling is known and applied in different fields such as image processing [19] and sensor networking [20], [21]. In [19], the technique is used for interactive virtual reality. For the same level of perceptual quality, the resolution required for a flat and curvy surface is different. The authors propose to adapt the resolution of rendering based on the degree of curvature of individual grids in the images. In sensor networking, the sensor nodes are often battery powered. Energy efficiency is a major technical issue to tackle. Raghunathan et al. [21] has pointed out work such as [20] has exploited the idea of adaptive sampling to conserve energy. Described in [20] is a sensor network that is deployed for environmental monitoring. To strike a balance in energy consumption and fidelity of the sensing results, they propose a multiscale sensing mechanism, in which a lower frequency/ density sensing is running continuously and the higher frequency/density sensing is triggered only when the events of interests are detected by the lower level. We also exploited the idea of adaptive sampling for energy-efficient boundary detection for location-aware applications.

\subsection{Location Estimation Techniques}

There are different techniques developed for range estimations. These techniques commonly require signal transmissions between the observer and the target observed. The major differences are the properties of calibration methods and the usage of signal sources. Our adaptive sampling mechanism is independent of the range estimation techniques and complementary in that the frequency of range estimations can be optimized for energy efficiency.

Most of the techniques use sonic, ultrasonic, and RF as signal sources. Given the assumption that signal propagates with constant velocity, time of arrival (TOA) is the most common method for estimating the distance by measuring the signal propagation time. Angle of arrival (AOA) is a network-based technique exploiting the geometric property of the arriving signal. By measuring the angle of the signal arrivals at more then one receiver, AOA is able to give a more precise location. Time difference of arrival (TDOA) [22] is also network based. It measures the time difference instead of the angle to infer distance. Some hybrid approaches of TOA, AOA, and TDOA are also proposed, and it is still an active research topic in the field of localization.

Another type of techniques measure the received RSSI. These techniques exploit the decaying model of electronicmagnetic field to translate RSSI to the corresponding distance [23], [24]. Also, the frequency bands used for transmission vary. For example, the well-known RADAR system [25] uses the RF. LADAR and SONAR use the visible light and the audible sound bands, respectively. RADAR, SONAR, and MoteTrack [3], for instances, analyze the signal reflected from the object to estimate location. A recent innovation, Cricket [26], takes a hybrid approach, using both the RF and ultrasonic bands.

Different from the signal-transmission-based methods, there exists another class of localization systems based on incremental motion from wearable sensors [27], [28]. These systems use accelerometers, digital compass, or orientation sensors to detect relative changes between locations. The energy consumption of the tag, thus, can be significantly lower than aforementioned localization systems. These localization systems are most useful for environment where building an infrastructure for localization is difficult. However, a major drawback of such systems is that the localization errors accumulate over time. In case of boundary detection applications where the target might need to be monitored continuously for a long time and the boundary detection error might need to be controlled within a certain limit, the localization systems based on estimating incremental positional changes will not be appropriate. In other words, the RF-based systems and accelerometer/gyroscope-based systems might not be very comparable in terms of boundary detection accuracy.

\subsection{Energy-Efficient Designs}

Communication has always been the main energy consumer in wireless systems, therefore, the energy-efficient issue has received lots of attentions [29]. This leads to low-power design within the physical layer in order to reduce the sources of power consumption within mobile terminals [30]. Adaptive error and power control are applied to energyefficient protocols within the MAC layer of wireless networks, and power-conserving protocols within the LLC layer [31], [32]. Power-aware protocols within the network layer exploit the trade-off between frequent topology updates (resulting in improved routing) and bandwidth consumed by increased update messages [33]. Opportunities for saving battery power within the transport layer lie in the sensitivity to wireless environment. Selective acknowledgments and explicit loss notification are used to handle losses. At application layer, techniques are developed specifically for different applications. Our work, the adaptive sampling mechanism, works on improving energy efficiency to support location-aware applications. 


\section{CONCLUSION AND Future Work}

In this work, we have proposed a mobility-aware adaptive sampling mechanism that can meet the accuracy requirements of applications but significantly reduce energy consumption in the underlying localization systems. Through simulations and live tests, we show that with the adaptive mechanism, we are able to enhance the energy efficiency of the locating system yet maintain the location information accuracy. The simulation results further suggest that the mechanism can actually adapt and benefit the most when the user is stationary. Although the mechanism performance is somehow adversely affected by positioning errors in the localization systems, we show the significant performance improvement can still be realized by the proposed adaptive sampling mechanism.

\section{ACKNOWLEDGMENTS}

The authors would like to thank the anonymous reviewers for their constructive comments. This work was supported in part by grants from the National Science Council of Taiwan under Contracts NSC 96-2220-E-002-016, NSC 962218-E-002-014, and NSC 96-2220-E-002-024, and the Digital Health Project from Intel Taiwan.

\section{REFERENCES}

[1] M. Hazas, J. Scott, and J. Krumm, "Location-Aware Computing Comes of Age," Computer, vol. 37, no. 2, pp. 95-97, 2004.

[2] M. Youssef and A.K. Agrawala, "Handling Samples Correlation in the Horus System," Proc. IEEE INFOCOM, 2004.

[3] K. Lorincz and M. Welsh, "MoteTrack: A Robust, Decentralized Approach to RF-Based Location Tracking," Personal and Ubiquitous Computing, vol. 11, no. 6, pp. 489-503, 2007.

[4] D. Madigan, E. Einahrawy, R.P. Martin, W.-H. Ju, P. Krishnan, and A.S. Krishnakumar, "Bayesian Indoor Positioning Systems," Proc. IEEE INFOCOM '05, pp. 1217-1227, 2005.

[5] K. Yedavalli, B. Krishnamachari, S. Ravula, and B. Srinivasan "Ecolocation: A Sequence Based Technique for RF Localization in Wireless Sensor Networks," Proc. Fourth Int'l Conf. Information Processing in Sensor Networks (IPSN '05), pp. 285-292, 2005.

[6] N. Ravi, P. Shankar, A. Frankel, A. Elgammal, and L. Iftode, "Indoor Localization Using Camera Phones," Proc. Seventh IEEE Workshop Mobile Computing Systems and Applications: Supplement (WMCSA '06), pp. 1-7, 2006.

[7] M.B. Kjærgaard, G. Treu, and C. Linnhoff-Popien, "Zone-Based RSS Reporting for Location Fingerprinting," Pervasive, pp. 316-333, 2007.

[8] L. Breslau, D. Estrin, K.R. Fall, S. Floyd, J.S. Heidemann, A. Helmy, P. Huang, S. McCanne, K. Varadhan, Y. Xu, and H. Yu, "Advances in Network Simulation," Computer, vol. 33, no. 5, pp. 59-67, 2000.

[9] D. Johnson and D. Maltz, "Dynamic Source Routing in Ad Hoc Wireless Networks," Mobile Computing, vol. 353, pp. 153-181, 1996.

[10] J. Hightower and G. Borriello, "Particle Filters for Location Estimation in Ubiquitous Computing: A Case Study," Ubicomp, pp. 88-106, 2004.

[11] J. Polastre, R. Szewczyk, and D.E. Culler, "Telos: Enabling UltraLow Power Wireless Research," Proc. Fourth Int'l Conf. Information Processing in Sensor Networks (IPSN '05), pp. 364-369, 2005.

[12] Chipcon: CC2420 802.15.4 Compliant Radio, http:// www.chipcon.com, 2008.

[13] ADXL202 Datasheet, http://www.analog.com/, Oct. 2000.

[14] ADXL330 Datasheet, http://www.analog.com/, Sept. 2006.

[15] S. Basagni, I. Chlamtac, V.R. Syrotiuk, and B.A. Woodward, "A Distance Routing Effect Algorithm for Mobility (Dream)," Proc. ACM MobiCom '98, pp. 76-84, 1998.

[16] A. Bhattacharya and S.K. Das, "Lezi-Update: An InformationTheoretic Framework for Personal Mobility Tracking in PCS Networks," Wireless Networks, vol. 8, no. 2/3, pp. 121-135, 2002.
[17] G. Wan and E.C. Lin, "A Dynamic Paging Scheme for Wireless Communication Systems," Proc. ACM MobiCom '97, pp. 195-203, 1997.

[18] Y. Xu, J. Winter, and W.-C. Lee, "Prediction-Based Strategies for Energy Saving in Object Tracking Sensor Networks," Proc. IEEE Int'l Conf. Mobile Data Management (MDM '04), pp. 346-357, 2004.

[19] G. Debunne, M. Desbrun, M.-P. Cani, and A.H. Barr, "Dynamic Real-Time Deformations Using Space and Time Adaptive Sampling," Proc. ACM SIGGRAPH' 01, pp. 31-36, 2001.

[20] M.A. Batalin, M.H. Rahimi, Y. Yu, D. Liu, A. Kansal, G.S. Sukhatme, W.J. Kaiser, M.M. Hansen, G.J. Pottie, M.B. Srivastava, and D. Estrin, "Call and Response: Experiments in Sampling the Environment," Proc. Second ACM Conf. Embedded Networked Sensor Systems (SenSys '04), pp. 25-38, 2004.

[21] V. Raghunathan, S. Ganeriwal, and M. Srivastava, "Emerging Techniques for Long Lived Wireless Sensor Networks," IEEE Comm. Magazine, vol. 44, no. 4, pp. 108-114, 2006

[22] L. Cong and W. Zhuang, "Hybrid TDOA/AOA Mobile User Location for Wideband CDMA Cellular Systems," IEEE Trans. Wireless Comm., vol. 1, no. 3, pp. 439-447, July 2002.

[23] N. Patwari, I. Hero, A.O. , M. Perkins, N. Correal, and R. O'Dea, "Relative Location Estimation in Wireless Sensor Networks," IEEE Trans. Signal Processing vol. 51, no. 8, pp. 2137-2148, Aug. 2003.

[24] D. Niculescu, "Positioning in Ad Hoc Sensor Networks," IEEE Network, vol. 18, no. 4, pp. 24-29, 2004.

[25] P. Bahl and V.N. Padmanabhan, "Radar: An In-Building RF-Based User Location and Tracking System," Proc. IEEE INFOCOM '00, pp. $775-784,2000$.

[26] N.B. Priyantha, A. Chakraborty, and H. Balakrishnan, "The Cricket Location-Support System," Proc. ACM MobiCom '00, pp. 32-43, 2000.

[27] S.-Y. Yeh, K.-H. Chang, C.-I. Wu, H.-H. Chu, and J.Y.-J. Hsu, "Geta Sandals: A Footstep Location Tracking System," Personal and Ubiquitous Computing, vol. 11, no. 6, pp. 451-463, 2007.

[28] S.-W. Lee and K. Mase, "Incremental Motion-Based Location Recognition," Proc. Fifth Int'l Symp. Wearable Computers (ISWC '01), p. 123, 2001.

[29] C.E. Jones, K.M. Sivalingam, P. Agrawal, and J.-C. Chen, "A Survey of Energy Efficient Network Protocols for Wireless Networks," Wireless Networks, vol. 7, no. 4, pp. 343-358, 2001.

[30] A.P. Chandrakasan and R.W. Brodersen, Low Power Digital CMOS Design. Kluwer Academic Publisher, 1995.

[31] S. Singh and C.S. Raghavendra, "Pamas-Power Aware MultiAccess Protocol with Signalling for Ad Hoc Networks," SIGCOMM Computer Comm. Rev., vol. 28, no. 3, pp. 5-26, 1998.

[32] K.M. Sivalingam, J.-C. Chen, P. Agrawal, and M.B. Srivastava, "Design and Analysis of Low-Power Access Protocols for Wireless and Mobile ATM Networks," Wireless Networks, vol. 6, no. 1, pp. 73-87, 2000.

[33] L.M. Feeney, "An Energy Consumption Model for Performance Analysis of Routing Protocols for Mobile Ad Hoc Networks," Mobile Networks and Applications, vol. 6, no. 3, pp. 239-249, 2001.

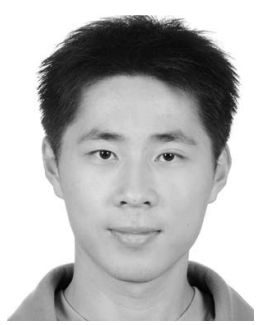

Tsung-Han Lin received the BS degree in electrical engineering from National Taiwan University, Taipei, in 2005. He is currently a master's student in the Department of Electrical Engineering, National Taiwan University. His research interests include sensor networking and wireless networking. $\mathrm{He}$ is a student member of the IEEE and the ACM. 


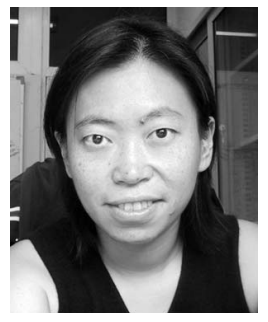

Polly Huang received the BS degree in mathematics from National Taiwan University (NTU), Taipei, in 1993 and the MS and PhD degrees in computer science from the University of Southern California, in 1997 and 1999, respectively. In February 2003, she joined the Department of Electrical Engineering, NTU, where she currently holds an associate professor position. Prior to joining NTU, she worked as a postdoctoral research scientist in the Computer Engineering and Networks Laboratory (TIK), Swiss Federal Institute of Technology (ETH) Zurich and as a postdoctoral fellow in the Institute of Pure and Applied Mathematics, UCLA. Her research interests include sensor networking, multimedia networking, and Internet characterization. She serves currently on the editorial board of the Journal of Communications and Networks. She is a member of the IEEE and the ACM.

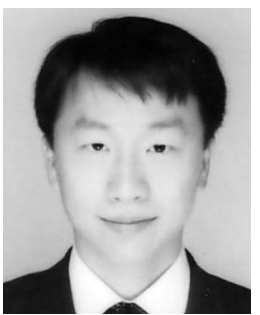

Hao-Hua Chu received the BS degree in computer science from Cornell University, in 1994 and the PhD degree in computer science from the University of Illinois at UrbanaChampaign, in 1999. He is an associate professor in the Graduate Institute of Networking and Multimedia and Department of Computer Science and Information Engineering, National Taiwan University, Taipei. From 1999 to 2000 , he was a senior software engineer at Intel. From 2000 to 2003, he was a project manager at (NTT) DoCoMo USA Labs. His research interests include pervasive computing and sensor/wireless network. $\mathrm{He}$ is a member of the IEEE and the ACM.

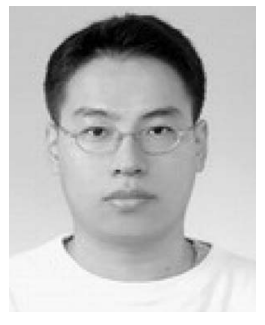

Chuang-Wen You received the BS degree from National Chiao Tung University, in 2002 and the $\mathrm{PhD}$ degree from National Taiwan University, in 2007. He is a senior software engineer at ubitus Inc., Taipei. His research interests include the broad area of distributed systems or sensor networks.

$\triangleright$ For more information on this or any other computing topic, please visit our Digital Library at www.computer.org/publications/dlib. 